\title{
THE WIENER LEMMA AND COCYCLES
}

\author{
MAREK RYSZARD RYCHLIK
}

(Communicated by Kenneth Meyer)

\begin{abstract}
We give a sufficient and necessary condition for a function with its values in the unit circle to be a multiplicative coboundary. This theorem generalizes the following result of Veech [1]. Let $T: \mathbf{T} \rightarrow \mathbf{T}$ be a rotation of the unit circle $\mathbf{T}$ by an irrational angle $\theta$. Let $F: \mathbf{T} \rightarrow \mathbf{T}$ be a measurable function. Then $F$ is a multiplicative coboundary iff
\end{abstract}

$$
\int_{\mathbf{T}} F(x) F(T x) \cdots F\left(T^{n-1} x\right) d \mu(x) \rightarrow 1, \quad \text { as }\|n \theta\| \rightarrow 0,
$$

where $\|n \theta\|$ is the distance of $n \theta$ from integers and $\mu$ is the Haar measure.

Let $T:(X, \Sigma, \mu) \rightarrow(X, \Sigma, \mu)$ be a measure preserving automorphism which is ergodic. Let $F: X \rightarrow \mathbf{T}$ be a measurable function. We call $F$ a coboundary $\bmod \lambda$ if there is a function $g: X \rightarrow \mathbf{T}$ and $\lambda \in \mathbf{T}$ such that $F(x)=\lambda g(x) / g(T x)$ a.e. For every integer $n \geq 0$, let $F_{n}(x)=F(x) F(T x) \cdots F\left(T^{n-1} x\right)$. For $n<0$ we set $F_{n}(x)=F_{-n}\left(T^{n} x\right)$.

DEFinition. Let $A \subset \mathbf{Z}$. The upper density of $A$ is the number

$$
\varlimsup_{n \rightarrow \infty} \frac{1}{2 n+1} \operatorname{card}([-n, n] \cap A) .
$$

THEOREM 1. The following two conditions are equivalent:

(i) $F$ is a coboundary $\bmod \lambda$.

(ii) There is a function $f \in L^{2}(X, \Sigma, \mu)$ and $\varepsilon>0$ such that the set

$$
\left\{n \in \mathbf{Z}:\left|\int_{\mathbf{T}} F_{n}(x) f\left(T^{n} x\right) \overline{f(x)} d \mu(x)\right|>\varepsilon\right\}
$$

has positive upper density.

PROOF. This theorem is an easy corollary of Wiener's Theorem. Let

$$
U: L^{2}(X, \Sigma, \mu) \rightarrow L^{2}(X, \Sigma, \mu)
$$

be a unitary operator defined as follows:

$$
U f(x)=F(x) f(T x) .
$$

Then the condition of being a coboundary $\bmod \lambda$ is equivalent to the existence of $g \in L^{2}(X, \Sigma, \mu)$ such that $U g=\lambda g$. In fact, if such $g$ exists then $|g \circ T|=|g|$ and by ergodicity the modulus of $g$ is constant a.e., so we can assume that it is equal to 1 .

Received by the editors November 2, 1987.

1980 Mathematics Subject Classification (1985 Revision). Primary 58F 11.

Key words and phrases. Wiener lemma, multiplicative cocycles. 
We invoke Wiener's Theorem now (see [2]):

$$
\frac{1}{2 n+1} \sum_{k=-n}^{n}\left|\left(U^{k} f, f\right)\right|^{2} \rightarrow \sum_{\lambda \in \mathbf{T}}\left|\sigma_{f}(\{\lambda\})\right|^{2}
$$

where $\sigma_{f}$ is the spectral measure of $f$. This means that the set of the eigenvalues of $U$ is nonempty iff for some $\varepsilon>0$ and some $f \in L^{2}(X, \Sigma, \mu)$ the set $\{n \in$ $\left.\mathbf{Z}:\left|\left(U^{n} f, f\right)\right|>\varepsilon\right\}$ has positive upper density. This is exactly condition (ii).

COROLLARY. Suppose there is an $\varepsilon>0$ such that the set:

$$
\left\{n \in \mathbf{Z}:\left|\int_{\mathbf{T}} F_{n}(x) d \mu(x)\right|>\varepsilon\right\}
$$

has positive upper density. Then $F$ is a coboundary $\bmod \lambda$.

ProOF. We substitute $f=1$ in condition (ii) and use the fact that $F_{n}=$ $U^{n} 1$.

Now let $T$ be just a rotation of $\mathbf{T}$ by an irrational angle $\theta$. Veech [1] proved the following result (unfortunately, this is the only application of Theorem 1 known to the author).

THEOREM 2. The function $F: \mathbf{T} \rightarrow \mathbf{T}$ is a coboundary (mod 1) iff

$$
\int_{\mathbf{T}} F_{n}(x) d \mu(x) \rightarrow 1, \quad \text { as }\|n \theta\| \rightarrow 0 .
$$

PROOF. We are going to derive this theorem from Theorem 1. It is easy to show that if $F$ is a coboundary then (iii) holds. The other implication can be proved as follows. From (*) we know that for some $\delta>0\|n \theta\|<\delta$ implies $\left|\int F_{n}(x) d \mu(x)\right|>1 / 2$. The set of $n$ such that $\|n \theta\|<\delta$ has positive upper density. Therefore the assumptions of Theorem 1 are satisfied and $F$ is a coboundary $\bmod \lambda$. It is easy to see that $(*)$ forces $\lambda=1$.

Theorem 1 generalizes without many changes to the actions of $\mathbf{Z}^{n}$ on $X$. We would like to conjecture that this theorem generalizes to the actions of amenable groups.

The author greatly appreciates helpful conversations with D. Lind, K. Merill and E. Glasner at the University of Washington.

\section{REFERENCES}

1. W. A. Veech, Strict ergodicity in zero dimensional dynamical systems and the Kronecker-Weyl theorem mod 2, Trans. Amer. Math. Soc. 140 (1969), 1-33.

2. I. P. Kornfel'd, S. V. Fomin and Ya. G. Sinai, Ergodic theory, Springer-Verlag, New York, 1982.

School of Mathematics, Institute for Advanced Study, Princeton, New JERSEY 08540 